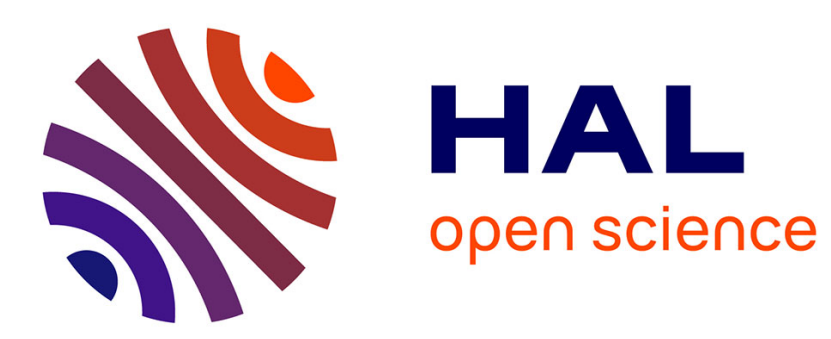

\title{
De l'examen du patrimoine littéraire français à la consécration des contemporains : le cas du XVIIe siècle
}

Emmanuelle Mortgat-Longuet

\section{To cite this version:}

Emmanuelle Mortgat-Longuet. De l'examen du patrimoine littéraire français à la consécration des contemporains : le cas du XVIIe siècle. Revue d'histoire littéraire de la France, 2013, 113 (3), pp.527544. 10.3917/rhlf.133.0527 . hal-01407199

\section{HAL Id: hal-01407199 \\ https://hal.parisnanterre.fr/hal-01407199}

Submitted on 17 Feb 2021

HAL is a multi-disciplinary open access archive for the deposit and dissemination of scientific research documents, whether they are published or not. The documents may come from teaching and research institutions in France or abroad, or from public or private research centers.
L'archive ouverte pluridisciplinaire HAL, est destinée au dépôt et à la diffusion de documents scientifiques de niveau recherche, publiés ou non, émanant des établissements d'enseignement et de recherche français ou étrangers, des laboratoires publics ou privés. 


\title{
DE L'EXAMEN DU PATRIMOINE LITTÉRAIRE FRANÇAIS À LA CONSÉCRATION DES CONTEMPORAINS : LE CAS DU XVII SIÈCLE
}

\author{
Emmanuelle Mortgat-Longuet*
}

En 1685, au seuil de ses Jugemens des sçavans - vaste revue critique des auteurs les plus «considérables » de tous les temps -, Adrien Baillet raconte qu'il a douté longtemps s'il devait parler des vivants : paraphrasant Velleius Paterculus, il estime en effet que «c'est une espéce de badinerie de compter et de dépeindre ceux que nous avons présens devant les yeux, n'étant pas d'ailleurs aussi aisé de les censurer que de les admirer ». Il s'est pourtant déterminé à les insérer dans son ouvrage, persuadé que la "curiosité cherche encore plus à se satisfaire sur les vivans que sur les morts $^{1} »$.

Cette position paradoxale témoigne de certaines difficultés rencontrées par ceux qui, au XVII ${ }^{e}$ siècle, entendent présenter la production littéraire de leur temps comme le terme d'un plus large procès historique. Nombreux sont en effet à cette époque les bilans qui, sous des formes diverses (récits rétrospectifs, traités, séries de vies de poètes, histoires de tel ou tel genre, etc.), entendent retracer l'histoire d'une partie ou de l'ensemble des lettres françaises - ils se différencient en cela des divers commentaires ou multiples débats critiques au sein desquels l'approche des œuvres et des auteurs

* Université Paris-Ouest-Nanterre.

1. Nos références à l'ouvrage d'Adrien Baillet [1 ${ }^{\text {re }}$ éd. 1685-1686] se feront désormais dans l'édition posthume des Jugemens des savans [...] revîs, corrigez, et augmentez par Mr. de la Monnoye, Amsterdam, Aux depens de la Compagnie, 1725. Nous citons l'« Avertissement », t. I, vol. I, pp. XVI-XVII - sur cette question, voir encore les pp. 26, 27, 54, 110 du traité liminaire Des Jugemens sur les livres en général. (Voir Velleius Paterculus, Histoire romaine, II, II, XXXVI [éd. Joseph Hellegouarc'h, Paris, « Les Belles Lettres », 1982, p. 44].)

RHLF, 2013, n³, pp. 527-544 
est globalement plus synchronique - et certains de ces bilans témoignent $\mathrm{du}$ souci de prendre en compte la création contemporaine. Aussi leurs auteurs peuvent-ils voir dans cet interdit qui frappe d'exclusion les vivants en considération de bienséances humaines, sociales ou politiques, un risque d'appauvrissement : amputant d'au moins une partie leur objet d'étude, il minerait d'emblée toute tentative pour octroyer à l'actualité cette perspective historique. Or - et nous touchons là à l'une des tensions qui, au XVII ${ }^{\mathrm{e}}$ siècle, déterminent la question qui nous intéresse dans ce volume - pour des hommes qui estiment tous vivre une période cruciale de l'histoire (même si les partisans des anciens et ceux des modernes en ont, on le sait, une perception bien différente), quel sens y aurait-il à dresser des bilans qui n'éclaireraient d'une quelconque manière le présent ? Ainsi voit-on certains composer parfois avec cet interdit, le contourner, voire l'enfreindre, comme le fait Adrien Baillet, janséniste qu'on ne peut soupçonner pourtant du moindre dédain pour les bienséances : le principe d'exclusion des vivants est au moins autant invoqué que pragmatiquement adapté.

C'est sans doute aussi que cette « curiosité » pour les vivants observée par Baillet est signe d'un changement du rapport au temps, conduisant dorénavant à un intérêt de plus en plus aiguisé pour toute forme d'actualité ce qui, en matière de création littéraire, ne va pas sans poser problème : à cette époque, si l'on veut tenir registre des plus récentes productions et les inscrire dans le cours d'une histoire, on se heurte d'emblée à l'hypothèse de leur fragilité par rapport aux grands modèles vainqueurs du temps, au soupçon de leur sujétion à des modes éphémères ou encore à l'ombre que pourraient jeter les faiblesses intrinsèques de ceux qui entreprennent cette tâche. Ainsi ce mouvement qui va de l'examen du patrimoine littéraire à celui de la création contemporaine s'inscrit-il au cœur de différentes tensions entre les circonstances toujours suspectes de contingence dont il pourrait relever et sa prétention - même implicite - à travailler d'ores et déjà pour l'inéluctable instance qu'est encore alors, aux yeux de tous, la " postérité ». Pour rendre compte de ces tensions, nous n'entendons pas définir au préalable la notion de «contemporain » puisque, justement, cette définition et ses déterminations constituent un des enjeux de ces discours rétrospectifs : il s'agit plutôt d'en saisir les raisons. C'est pourquoi, après avoir relativisé la menace que l'opposition entre les morts et les vivants fait peser sur l'historicisation des contemporains, nous examinerons comment les différentes conceptions du temps conduisent à des découpages apparemment contradictoires du « présent ", de manière à pouvoir considérer ce qu'apportent à la production littéraire contemporaine et à ses acteurs les différentes modalités de leur inscription dans une histoire qui les englobe et les dépasse. 
À «l'âge de l'éloquence », les hommes de lettres ont en effet pour la plupart en mémoire les propos des anciens concernant la difficulté à parler des vivants, non seulement ceux de Velleius Paterculus, mais encore au moins ceux de Juvénal et de Cicéron. On sait que dans le Brutus, « véritable histoire critique de l'éloquence républicaine à $R_{0} \mathrm{e}^{2}$ » du reste mise à la disposition d'un public élargi par la traduction de Giry en 1652, Cicéron se dit résolu à ne nommer aucun vivant, notamment pour éviter, aux dires de Brutus, le trop grand nombre d'orateurs à évoquer et les plaintes que pourraient lui occasionner ses silences ou sa tiédeur - ce qui au demeurant n'empêche pas dans l'ouvrage de longues évocations de Marcellus et de César ${ }^{3}$. $\mathrm{Au} \mathrm{XVII}^{\mathrm{e}}$ siècle, même si les raisons évoquées revêtent dorénavant des significations différentes, la mort de ceux dont on souhaite parler est encore généralement perçue comme une étape propice à la pertinence du jugement historique et critique : en libérant notamment les censeurs de leurs " préjugés » liés à la qualité et à la dignité des auteurs, elle rend les ouvrages " égaux » comme le dit le janséniste Baillet ${ }^{4}$. Ainsi, dans de nombreux textes qui entendent rendre compte de la genèse et faire le bilan du patrimoine littéraire - quel qu'il puisse être -, la mort des hommes constitue-t-elle un élément déterminant de composition. C'est le cas par exemple de la Relation contenant l'histoire de l'Academie françoise que Pellisson rédige en 1652 et publie en 1653. Dans cet ouvrage, il dote la toute récente Académie, âgée d'à peine dix-huit ans, d'un passé : il fait des Académiciens des générations actuelle et tout juste précédente les acteurs d'une geste dont il juge utile de faire $l^{\prime}$ « histoire ${ }^{5} »$, conduisant celle-ci « jusqu'à présent ». Comme cette entreprise requiert notamment l'évocation des « excellents esprits ${ }^{6}$ » qui l'animent et dont beaucoup continuent de produire des ouvrages, Pellisson est conduit à scinder son recensement : d'un côté dix-sept morts à propos desquels il déclare pouvoir parler en " liberté ${ }^{7} »$ et dont les notices, quoique brèves, sont conformes à la tradition de la "vie ", et de l'autre quarante vivants qui, dans le « catalogue » qui leur est consacré, n'ont droit qu'à une rudimentaire phrase d'état civil, suivie d'un simple répertoire d'ouvrages ${ }^{8}$.

2. Marc Fumaroli, L'Âge de l'éloquence. Rhétorique et "res literaria » de la Renaissance au seuil de l'époque classique, Genève, Droz, 1980, p. 47.

3. Cicéron, Brutus, LXV, 231-LXXIII, 254 (éd. Jules Martha, Paris, " Les Belles Lettres », 1973, pp. 82-91).

4. Adrien Baillet, Des Jugemens sur les livres [...], op. cit., p. 54.

5. Paul Pellisson [puis l'abbé d'Olivet], Histoire de l'Académie française, éd. Charles-Louis Livet, Paris, Didier, 1858, t. I, p. 4 et 160 ; pp. 127 sq.

6. Ibid., p. 318 et p. 4 .

7. Ibid., p. 250.

8. Ibid., pp. 251-318. 
Seuls les morts, que leur disparition date de quelques semaines ou de plusieurs années, peuvent donc faire ici l'objet d'un discours rétrospectif et d'un jugement critique surplombant: c'est le cas par exemple de Du Chastelet dont l'œuvre apparaît traversée par le cours de l'histoire - Pellisson se faisant l'écho des grandes différences remarquées entre les ouvrages composés avant l'entrée à l'Académie et ceux qui en revanche auraient bénéficié des exercices de l'institution ${ }^{9}$-, alors que les œuvres de même date des vivants ne donnent lieu à aucun commentaire. C'est encore la mort des poètes qui semble ouvrir l'espace du « récit de vie », élément fondamental de la monumentale Histoire générale et particulière des poètes françois, anciens et modernes rédigée par l'Académicien Colletet, sous l'impulsion de Richelieu, entre 1635 et 1659. En faisant la « vie » de plus de quatre cents poètes du Moyen Âge à son époque, Colletet entend rendre hommage à ceux qui ont vaillamment illustré les potentialités d'une toute jeune langue française au sein d'une ample production bien imparfaite sans doute, mais riche d'avenir, et sa vaste fresque conduit évidemment à promouvoir les promesses des temps présents ${ }^{10}$. Pourtant, si l'on observe le travail consacré aux poètes les plus récents ${ }^{11}$, on s'aperçoit que dans la plus grande majorité des cas, Colletet a attendu la disparition des poètes pour en faire la notice, par exemple celles de Jean Besly mort en 1644 ou de François de Maynard mort en $1646^{12}$. En outre, c'est la date supposée du décès qui est généralement retenue par Colletet pour le classement chronologique qu'il prévoit pour ses vies ${ }^{13}$. En cela, ses notices gardent encore un lien avec le contexte institutionnel de la mort qui, jusqu'alors, présidait encore pour une bonne part à l'écriture des vies des «illustres ${ }^{14}$ ", même si par bien d'autres aspects, il s'en détache largement puisque dorénavant, dans le plan tripartite qu'il rend systématique au sein de ses notices, passant de l'homme à son œuvre puis à la réception de celle-ci, l'évaluation des ouvrages prend le pas sur le « discours de la personne ». Une limite semble donc a priori posée, à

9. Ibid., p. 169 (Paul Hay du Chastelet est mort en 1636). Voir encore le jugement sur Claude de Malleville (mort en 1647), p. 211.

10. On trouvera de plus amples développements et des indications bibliographiques sur cette entreprise de Colletet dans notre Clio au Parnasse. Naissance de l'" histoire littéraire " française aux XVI et $X V I I^{e}$ siècles, Paris, Champion, 2006, pp. 185-233 et 345-358, et dans " L'emploi du mot "vie" chez G. Colletet : de l'éloge de l'illustre à la critique du poète français ", Le Lexique métalittéraire (XVI ${ }^{e}$-XVII siècles), dir. Michel Jourde et Jean-Charles Monferran, Genève, Droz, août 2006, pp. 89-106.

11. Cette tâche n'est guère facilitée par les multiples infortunes qu'a subies le manuscrit dont nous ne connaissons plus, pour le dire brièvement, qu'à peu près la moitié.

12. Colletet fait en revanche la «vie » de François Champflour sans savoir s'il est ou non encore en vie.

13. Cette date figure généralement en tête de la notice, à côté du nom du poète.

14. Voir Daniel Ménager, "Théodore de Bèze, biographe de Calvin ", Bibliothèque d'Humanisme et Renaissance, t. XLV, 1983, $\mathrm{n}^{\circ} 2$, pp. 231-255. 
cette époque, à l'inscription dans les bilans historiques du plus extrême contemporain. Et même à la fin du siècle, alors que d'aucuns sont tout à chanter la gloire des temps présents, un Moderne comme Fontenelle se refuse, en 1692, à introduire les vivants dans son « histoire de la Poësie Françoise, par les ouvrages même des Poëtes », anthologie allant de Villon jusqu'à Benserade mort seulement « depuis trois mois » : c'est qu'un choix dans l'œuvre des vivants aurait été « une espece de jugement, auquel ils n'auroient apparemment pas souscrit. Il est permis d'en user plus librement avec les morts ${ }^{15} »$.

Est-ce à dire alors que la mort ferait passer l'œuvre du « temps de son auteur» au «temps de la postérité » et que sur ce point le moderne Fontenelle rejoindrait paradoxalement un Longepierre ou un Boileau, le premier jugeant en 1687 que nous sentons bien plus vivement le prix d'une chose " après que nous l'avons perduë », le second considérant en 1694 que trente ans suffisent à renverser une gloire comme celle de Balzac et qu' « il n'y a qu'une longue suite d'années qui puisse établir la valeur et le vrai merite d'un Ouvrage ${ }^{16} »$ ? Les hommes du XVII ${ }^{\mathrm{e}}$ siècle seraient-ils donc tous «Anciens » en ce point ? Pour se convaincre qu'il n'en est rien, on peut tout d'abord observer que les différences dans la manière de traiter les vivants ne recoupent pas nécessairement le clivage entre les partisans des anciens et ceux des modernes. Dans le Traité de l'origine des romans de 1670 , où un historique et une poétique du genre romanesque se mêlent à une réflexion sur le fabuleux, Huet termine son enquête passant par la fable orientale, les romans grecs et latins, puis les vieux textes en français par une évocation de L'Astrée et des romans modernes, notamment ceux de Mademoiselle de Scudéry qui vivra encore une bonne trentaine d'années ${ }^{17}$ - on ne peut pourtant pas suspecter le savant, malgré sa fréquentation des milieux galants, de " modernisme ». Ensuite, il est évident que nombre de ces bilans et historiques sont justement conçus et rédigés pour aboutir précisément à la promotion de certains vivants - nous allons y revenir. Enfin et surtout, cette distinction entre les morts et les vivants ne semble pas l'élément qui rende le plus significativement compte de la délimitation et du

15. Bernard Le Bovier de Fontenelle, Recueil des plus belles pieces des Poëtes François, Tant Anciens que Modernes, depuis Villon jusqu'à M. de Benserade, avec l'histoire de leur vie [...], Paris, Barbin, 1692, reproduit dans Fontenelle, Euvres complètes, éd. Alain Niderst, Paris, Fayard, 1989-2001, t. IX, pp. 187-266. Nous citons la «Préface », assurément de Fontenelle lui-même, pp. 190-192.

16. Hilaire Bernard de Longepierre, Discours sur les Anciens, Paris, P. Aubouin, P. Emery et Ch. Clousier, 1687, p. 124 ; Nicolas Boileau, « Réflexion VII » des Réflexions critiques sur quelques passages du rhéteur Longin, Euvres complètes, éd. Françoise Escal, Paris, Gallimard, « La Pléiade », 1966, p. 524.

17. Pierre-Daniel Huet, Traité de l'origine des romans, éd. Camille Esmein, dans Poétiques du roman [...], Paris, Champion, 2004, pp. 359-535 (pp. 530-535 notamment). 
traitement de la création contemporaine. Reprenons l'exemple de l'Histoire de l'Academie françoise: les morts dont Pellisson assure parler avec « liberté » ne sont-ils pas encore présentés dans une certaine mesure comme des contemporains ? Ne pourraient-ils encore participer de l'actualité littéraire ? L'historien précise effectivement les liens factuels qu'ils ont encore avec le présent - il mentionne leurs relations avec des Grands toujours en place -, ou évoque leur survie éditoriale - il note que certains textes suscitent encore des débats ou doivent bientôt paraître ${ }^{18}$. On se doute que les propos flatteurs de Pellisson cherchent surtout à faire profiter le « Corps » de l'Académie de cette actualité symbolique des fondateurs disparus ${ }^{19}$ : leurs œuvres en illustreraient et pérenniseraient la vocation, contribuant à faire croire " encore aujourd'hui », comme elles le font pour lui ${ }^{20}$, que la langue française est «capable de toutes choses ». D'autre part, on peut encore observer que les remarques incidentes disséminées dans la plupart des vies en série de l'Histoire des poètes françois de Colletet forment une trame discursive générale - n'étant pas du reste sans évoquer celle que l'on trouve dans les Vies de Vasari, un des modèles probables de Colletet - qui constitue au bout du compte un discours circonstancié non seulement sur la création littéraire en général, mais aussi sur celle de son temps en particulier. En effet, de nombreux parallèles et bilans partiels esquissent un tableau du temps présent: évocation des résistances aux principes malherbiens (« Robert Angot»), triomphe du théâtre moderne (« Jean de La Peruse »), description des effets pervers du clientélisme et de l'insuffisance des droits d'auteur (« François de Belleforest ») $)^{21}$, etc. - bref, autant d'actualisations de son enquête sur les auteurs du passé, même ancien.

Ainsi, si la mort continue encore souvent à conditionner - conformément, notamment, à la tradition biographique -, l'entrée de l' « homme », en l'occurrence auteur d'une œuvre, dans les divers bilans et récits rétrospectifs, il n'empêche que, événement somme toute fortuit, cette disparition ne coïncide pas nécessairement avec la détermination d'un temps présent que les sens ou certains aspects de cette œuvre contribueraient, aux yeux de

18. Paul Pellisson, op. cit., par exemple les pp. 213-220, 230-232, 241-245 et les pp. 174, 180, 234-236, 250.

19. Paul Pellisson écrit : «[...] c'est ici l'Histoire de l'Académie, et non pas celle des Académiciens, dont, à vrai dire, je ne dois parler qu'autant qu'il est nécessaire pour faire juger de tout le Corps par quelques-uns de ses membres », ibid., p. 162.

20. Il le dit à propos d'un ouvrage de Jean Sirmond et de quelques autres, ibid., pp. 225-226 (voir aussi pp. 3-4 et 320).

21. Guillaume Colletet, Vie du poète normand Robert Angot sieur de l'Éperonière, éd. Prosper Blanchemain, Société rouennaise de bibliophiles, Rouen, Espérance Cagniard, 1873, pp. 4-6 ; Vies [... des] Poëtes angoumoisins, éd. Ernest Gellibert des Seguins, Paris, A. Aubry, 1862, pp. 212216 ; Vies des poètes gascons, éd. Philippe Tamizey de Larroque, Paris, A. Aubry, 1866, pp. 51-52. 
quelques-uns, à faire vivre et caractériser. Il reste donc à comprendre comment peut être véritablement construite l'« actualité » littéraire.

\section{DE LA DIVERSITÉ DES PRÉSENTS}

Le sentiment qu'ont de nombreux hommes de l'époque de vivre une période importante de l'histoire des savoirs et de la culture s'étaie pour certains d'une assurance qui va croître au cours du siècle : celle du progrès. En effet, parallèlement à la permanence, pour un bon moment encore, d'une conception cyclique de l'histoire et - pour le dire brièvement - d'une perception du temps comme continuité des présents ${ }^{22}$, commence à se manifester ici ou là une représentation plus cumulative du temps ${ }^{23}$ que l'on fonde maintenant parfois, notamment, sur l'avancée que peuvent représenter les acquis scientifiques et techniques des temps modernes - on sait qu'à cet égard les travaux de Francis Bacon qui se diffusent dans toute 1'Europe marquent une étape capitale ${ }^{24}$. En ce qui concerne plus précisément le domaine de la langue et des lettres - qu'il soit isolé ou non au sein de l'ensemble des « sciences »- il commence à paraître possible à certains de concevoir une discontinuité, de constater des changements et donc bientôt de distinguer une nouvelle période riche de potentialités. Cette attitude se retrouve notamment dans certains propos enthousiastes accompagnant la création de l'Académie ${ }^{25}$ qui, même s'ils ne sont pas unanimes et s'ils témoignent, comme on l'a montré, de diverses tensions ${ }^{26}$, révèlent notamment

22. Sur les sens que la perception du temps peut donner au XVI ${ }^{\mathrm{e}}$ siècle aux divers bilans rétrospectifs, voir François Roudaut, «Quelques remarques pour une anthropologie de l'histoire littéraire au XVI ${ }^{\mathrm{e}}$ siècle ", L'Histoire littéraire à l'aube du XXI siècle. Controverses et consensus, dir. Luc Fraisse, Paris, Puf, 2005, pp. 44-53.

23. Sur cette conception du progrès et ses antécédents, voir notamment Franco Simone, « Une entreprise oubliée des humanistes français. De la prise de conscience historique du renouveau culturel à la naissance de la première histoire littéraire ", Humanism in France [...], ed. by Anthony Herbert Tigar Levi, Manchester University, 1970, pp. 106-131 ; Claude Faisant, Mort et résurrection de la Pléiade (1585-1828), publ. par Josiane Rieu et alii, Paris, Champion, 1998, pp. 70-77 ; Jean Lecointe, L'Idéal et la différence [...], Genève, Droz, 1993, pp. 172-181.

24. Voir notamment Francis Bacon, science et méthode, dir. Michel Malherbe et Jean-Marie Pousseur, Paris, Vrin, 1985 ; L'Héritage baconien au XVII et au XVIII siècles, dir. Chantal Jaquet, Paris, Kimé, 2000 ; Didier Deleule, Francis Bacon et la réforme du savoir, Paris, Hermann, 2010 ; Chantal Jaquet, Bacon et la promotion des savoirs, Paris, Puf, 2010.

25. C'est ainsi que Colletet assure, en 1636, au cours de son Discours de l'Eloquence et de l'Imitation des Anciens que «[...] nous sçavons des choses qui leur [aux anciens] ont esté cachées, que le Temps nous a découvertes, et qu'il semble n'avoir réservées que pour nous. [...] la vieillesse du temps [,] peut donner aux hommes des connoissances, et des lumieres que l'enfance du monde ne leur pouvoit pas donner encore ", L'Art Poëtique [...], Paris, A. de Sommaville et L. Chamhoudry, 1958, p. 47.

26. Voir Roger Zuber, Les "Belles Infidèles » et la formation du goût classique [1 $1^{\mathrm{re}}$ éd. 1968], Paris, A. Michel, 1995, pp. 52-63, 81-96, 118-129 (notamment pp. 93-95). 
qu'on a pris acte des récentes évolutions de la langue et des conséquences que celles-ci peuvent avoir sur la production littéraire. C'est dans ce contexte qu'apparaissent les premières tentatives pour circonscrire et célébrer la grandeur du présent en matière littéraire, tentatives qui semblent sans doute d'autant plus nécessaires que toute inquiétude quant à l'avenir n'est pas bannie - soit que persiste le sentiment de la fragilité des choses, soit que l'on juge la langue non encore fixée «à son point de perfection », soit que l'on perçoive déjà à quel point le progrès risque de relativiser, à terme, les succès du présent ${ }^{27}$. Quoi qu'il en soit, cette inquiétude n'empêche pas la promotion du contemporain d'être tout particulièrement le fait non seulement des milieux proches de l'Académie et du pouvoir, mais encore, un peu plus tard, des cercles galants et enfin, bien sûr, dans le dernier quart du siècle, de ceux que l'on appellera bientôt les Modernes et qui témoignent d'une nouvelle forme de " présentisme ${ }^{28}$ ».

Comment donc peut être déterminé ce présent ? À cet égard, s'esquissent au cours du siècle deux mouvements apparemment contradictoires, l'un consistant à rendre historique un corpus littéraire de plus en plus récent, l'autre à faire en revanche reculer dans le passé les bornes de l'actualité. D'une part, en effet, ceux que l'on considère comme les acteurs d'un nouvel âge de l'histoire des lettres peuvent être extrêmement récents. Le premier découpage notable du «contemporain » au XVII ${ }^{\mathrm{e}}$ siècle est bien connu parce qu'il a eu, on le sait, une grande postérité : « Enfin Malherbe vint. »C'est pourtant très précocement, au cours des années 1630 , et probablement moins de cinq ans après la mort du poète, que la plupart des éléments de cette périodisation sont déjà en place, comme cela a pu être montré ${ }^{29}$. En effet, dans une lettre à Silhon, Balzac ne se borne pas à déplacer le schéma de la Renaissance après avoir évoqué les origines de la poésie française ${ }^{30}$, mais il estime que Malherbe est le point d'origine de l'ère présente parce qu'il a œuvré avec raison et goût : « Grâce à son jugement [...] il a formé et corrigé le génie de ses contemporains ; [...] C'est donc à nul autre [...] que

27. Colletet n'est pas isolé lorsqu'il affirme, pas plus d'une quinzaine d'années après la création de l'Académie : « Mais peut-être, tout parfaits que nous pensons être, la postérité nous traitera un jour comme nous traitons ceux qui nous ont devancés » (Vie d' « Antoine Heroët », Antoine Héroet, Euvres poétiques, éd. Ferdinand Gohin, Paris, Cornély et C ${ }^{\mathrm{ie}}$, 1909, pp. 155-156).

28. Voir notamment les analyses récentes de Larry Norman, The Shock of the Ancient, Chicago, The University of Chicago Press, 2011.

29. Nous nous appuyons ici sur les analyses de Claude Faisant, « Lieux communs de la critique classique et post-classique », Études françaises, XIII, 1-2, avril 1977, pp. 143-162.

30. «Le premier, Malherbe, ou parmi les premiers, vit la voie menant au poème ; il s'avança, parmi l'obscurité de l'erreur et de l'ignorance, vers la véritable lumière en tenant le premier les yeux fixés sur elle » («Lettre latine à Silhon » - qui daterait de 1633 -, dans Jean-Louis Guez de Balzac, [Livre unique d']Épîtres latines, trad. et éd. par Jean Jehasse et Bernard Yon, Université de Saint-Étienne, 1982, p. 106). 
sont le plus redevables nos lettres d'aujourd'hui, en langue vulgaire ${ }^{31}$. » Cette conception d'une fondation du présent par la raison et le goût malherbiens structure la représentation de l'histoire des lettres françaises tout au long du siècle, bien avant même le retentissement que Boileau peut lui donner. Pourtant, tous ne souhaitent pas s'en tenir là et certains, dans différents lieux ou cercles, préfèrent manifestement pondérer la rupture qu'il incarne pour promouvoir au sein de leurs bilans les hommes de la génération suivante. C'est le cas, entre autres ${ }^{32}$, de Pellisson, dorénavant de plus en plus influent dans la vie littéraire. Dans une lettre latine de 1655 destinée à conseiller un savant Anglais, Pellisson découpe l'histoire des lettres françaises selon trois périodes au sein desquelles il passe en revue les principaux domaines de production et dont la dernière court de Louis XIII et de Richelieu à son propre temps. Après hésitation, Pellisson choisit de repousser Malherbe dans l' «âge plus ancien », tant en ce qui concerne l'éloquence et les lettres « urbaines » que la poésie, ce qui lui permet, en affirmant que pour ces domaines « tout est presque de notre siècle », de rendre hommage à un grand nombre d'auteurs extrêmement récents ou parfaitement contemporains, dont beaucoup, qu'ils soient poètes mondains, hommes de religion, érudits, etc. sont ses confrères de l'Académie ou des membres du salon de Mademoiselle de Scudéry qu'il fréquente assidûment ${ }^{33}$. Ainsi Pellisson entend-il montrer qu'en France l'âge de Malherbe a laissé place à un nouvel épanouissement de lettres fortes et polies - et du reste un an plus tard, il fait clairement de Voiture qui «vint» renouveler la poésie, puis de Sarasin, les incarnations du génie du siècle présent ${ }^{34}$. Ces représentations n'ont pas perduré, on le sait. Mais elles montrent non seulement qu'en moins de vingt ans l'origine du temps présent a pu glisser d'une génération à une autre et le décalage chronologique presque s'abolir, mais encore que ce découpage du présent littéraire repose ici sur la perception d'une nouveauté des goûts et des pratiques ${ }^{35}$. Ces nouveaux découpages ne conduisent pas nécessairement, cela dit, à faire fi des acquis que représentent les aînés. Dans sa

31. Ibid., pp. 106-107. Nous soulignons.

32. Remarquons en outre que des textes d'une tout autre facture ou traversés par de tout autres desseins que ces bilans rétrospectifs peuvent également témoigner d'une promotion des plus récents contemporains (voir notamment les exemples analysés par Alain Génetiot, Le Classicisme, Paris, Puf, 2005, pp. 17-20).

33. « Lettre latine de Pellisson à Smith » [1655], dans William Henry Barber, « A Seventeenthcentury view of french literature », French Studies, Oxford, Basil Blackwell, 1948, vol. II, pp. 140147 (notamment pp. 145-147). Thomas Smith est membre du Christ's College de Cambridge et bibliothécaire de l'Université.

34. Paul Pellisson, Discours sur les Euvres de M. Sarasin, dans L'Esthétique galante [...], éd. dir. Alain Viala, 1989, pp. 69-72.

35. On trouvera de plus amples développements et indications bibliographiques sur ces périodisations et ces sélections, notamment au sein des textes cités de Balzac et de Pellisson, dans Clio au Parnasse, op. cit., pp. 174-177 et 268-276. 
Bibliothèque françoise de 1667, au sein du chapitre « Du Progrez de la langue françoise et des autheurs qui ont écrit en chaque Siecle » qui retrace l'histoire de la prose du Moyen Âge à son époque, Sorel fait également reculer Malherbe dans un «siècle » précédent, aux côtés de Du Vair, Du Perron et D'Urfé. Mais il ne refuse pourtant pas de voir à l'œuvre dans le présent les bénéfices du passé. Évoquant le «profit » retiré par ceux qui allaient écouter Malherbe, Sorel conclut que « la pluspart des bons Escrivains d'aujourd'huy ont esté de ce nombre, ou sont les Disciples de ses Disciples $\|^{36}$. La qualité de la production contemporaine n'est donc pas sans résulter de l'apport malherbien - l'ambivalence que celui-ci suscite dorénavant, obligeant à affiner le propos, conduit à différencier les plus récentes générations des précédentes non plus selon un simple principe de succession, de dépassement ou de recul, mais selon celui, même encore embryonnaire ici, d'un engendrement.

Cette propension à enregistrer et promouvoir les plus récents auteurs au terme des différents parcours rétrospectifs fragilise cependant tout découpage et rend logiquement nécessaires les mises à jour. Il faut dire en outre qu'est croissante l'appétence pour l'actualité d'un public désormais à l'affût de tout ce qui pourrait constituer son « évangile du jour ${ }^{37}$ » et que ces bilans se donnent à lire dans un contexte où toute information récente est précieuse, où les nouvelles se périment rapidement et où bientôt se multiplient les périodiques ${ }^{38}$. Ainsi Sorel entreprend-il en 1667 de réactualiser le catalogue des Académiciens dressé par Pellisson en 1652, en précisant que seule l'impression a mis un point d'arrêt à son travail ${ }^{39}$. S'il semble donc possible de définir en amont le temps présent par des charnières plus ou moins stables, en aval le temps semble en revanche toujours ouvert, comme si l'on attendait malgré tout encore le jugement rétrospectif de la postérité pour en arrêter les contours et décider des valeurs. Il ne semble pas qu'à cette époque la consécration des contemporains s'accompagne d'une quelconque émancipation du jugement des générations futures, mais bien plutôt que l'on s'efforce de le pressentir et de le faire sien : la postérité reste le tribunal intériorisé de ce temps présent.

36. Charles Sorel, La Bibliothèque françoise, $2^{\mathrm{e}}$ éd. augmentée, Paris, Les Libraires du Palais, 1667, pp. 260-261.

37. Nous renvoyons ici à l'introduction de Karen Abiven et Laure Depretto, «"L'Évangile du jour" : écrire l'actualité (XVI ${ }^{\mathrm{e}}-\mathrm{XVIII}{ }^{\mathrm{e}}$ siècles) », pour le numéro qu'elles ont dirigé sur ce sujet, Littératures classiques, ${ }^{\circ}$ 78, 2012, pp. 5-15. L'expression citée est de Madame de Sévigné.

38. Voir Jean Sgard, « La multiplication des périodiques », Histoire de l'édition française, dir. Roger Chartier et Henri-Jean Martin, Paris, Fayard, 1990 [1 $1^{\text {re }}$ éd. 1984], t. II, pp. 246-255, qui cite notamment cette déclaration du Journal des savants s'annonçant en 1665 comme hebdomadaire, " parce que les choses vieilliroient trop si l'on differoit d'en parler pendant l'espace d'un an ou d'un mois [...]» (p. 247).

39. Charles Sorel, op. cit., pp. 267-269. 
Parallèlement à cette propension à consacrer un présent de plus en plus récent ou immédiat, d'autres bilans s'efforcent au contraire de représenter une période contemporaine plus large et ce, d'autant plus que l'on avance dans le siècle. Quelques auteurs qui pouvaient déjà être rejetés dans un passé plus ou moins révolu sont à nouveau présentés comme des auteurs contemporains. Ainsi voit-on se construire un « siècle » largement accueillant voire, comme cela a été montré, constitué d'《amalgames » faisant coexister fallacieusement plusieurs générations ${ }^{40}$ - il y a de ce point de vue-là hésitations et concurrences entre des représentations et découpages du présent selon un ordre d'ores et déjà proprement littéraire et la tendance en pleine régénérescence à faire coïncider ce « siècle », comme le fait Perrault, avec l'ordre politique, notamment en le rattachant à la figure du « prince $»^{41}$. Ces constructions d'une période contemporaine élargie sont sans doute propices à une vision plus synthétique des événements, tendant à mettre en relief ce qui peut en résulter ou encore la valeur symbolique qu'on entend leur conférer. Sans aller jusqu'au cas-limite de la cartographie que pourrait par exemple représenter La Nouvelle Allégorique de Furetière ${ }^{42}$, on peut penser au tableau qu'en 1674, dans son Théâtre françois, Chappuzeau dresse de la vie théâtrale de son temps : si cet ouvrage prétend constituer « un petit corps d'Histoire $\aleph^{43}$ et s'il n'exclut pas la chronique souvent minutieuse des faits, il saisit ceux-ci en partie dans la relative fixité d'un aboutissement ${ }^{44}$, en regard de la situation d'exception à laquelle ils ont pu, d'après lui, donner lieu. Ainsi, animé comme bien d'autres par la conscience aiguë de l'exceptionnelle vitalité théâtrale de son époque, Chappuzeau fait-il remonter à cinquante ans le début de cette période dont il entend rendre compte. Se

40. Voir les analyses de Bernard Magné, Crise de la littérature française sous Louis XIV: humanisme et nationalisme, Atelier des thèses de Lille III-Diffusion Paris, Champion, 1976, t. I, pp. 20-26. Ces regroupements fallacieux semblent le lot de la construction des "grands siècles » (voir Roger Zuber, «Les "siècles" classiques », Les Émerveillements de la raison, Paris, Klincksieck, 1997, pp. 199-194).

41. Sur les évolutions des éléments constitutifs du concept de siècle et pour de plus amples indications bibliographiques, nous nous permettons de renvoyer à notre article: «Du "siècle d'Auguste" au Siècle de Louis XIV : quelques réflexions sur le concept de "siècle" du début du $\mathrm{XVII}^{\mathrm{e}}$ siècle à Voltaire ", Voltaire et le Grand siècle, dir. Jean Dagen et Anne-Sophie Barrovecchio, Oxford, The Voltaire Foundation, SVEC, 2006, pp. 97-116.

42. Antoine Furetière, La Nouvelle Allégorique [...] [1 $1^{\mathrm{re}}$ éd. 1658], éd. Mathilde Bombart et Nicolas Schapira, Toulouse, Société de Littératures classiques, 2004. Furetière fait remonter l'état des lieux qu'il dresse à l'époque de Richelieu. Sur le modèle cartographique et ses effets, voir Delphine Denis, Le Parnasse galant. Institution d'une catégorie littéraire au XVII siècle, Paris, Champion, 2001, pp. 21-36.

43. Samuel Chappuzeau, Le Théâtre françois, éd. Christopher J. Gossip, Tübingen, Gunter Narr Verlag, 2009, p. 54.

44. Voir par exemple la manière dont l'histoire de la Troupe du Marais et celle de la troupe du Palais-Royal sont perçues au travers du lustre présent de la « Troupe du Roy » qui les a réunies (ibid., pp. 183-193). 
justifiant de ne pas s'étendre sur les origines lointaines de la comédie, il écrit : "Cela n'a rien de commun avec notre siècle, et il me suffit de montrer de quelle manière se conduisent présentement les comédiens, et quelle est la nature de la comédie depuis qu'elle est dans son lustre par l'estime qu'en a fait un Armand de Richelieu, et les grâces que lui a données un Pierre Corneille $e^{45}$. » C'est aussi que cette durée de l'époque contemporaine, dont il fait remonter l'origine à Corneille et à Richelieu, permet de présenter la production cornélienne dans toute sa force - il est aidé en cela par la longévité du dramaturge qui va bientôt donner Suréna - et dès lors de présenter comme plus contingentes les dernières évolutions du goût incarnées par Quinault ou Racine qu'en bon pro-cornélien il déplore. Il est donc logique de trouver la même périodisation de l'époque contemporaine chez un autre partisan de Corneille, et non des moindres: Fontenelle. Dans son Histoire du théâtre françois et sa Vie de Corneille, qui datent de la dernière décennie du XVII ${ }^{e}$ siècle, il fait du dramaturge, contre un Racine qui a pourtant l'avantage de la postériorité et de plus récents succès, le «père » d'un théâtre français qu'il a porté à son point de perfection et un modèle, au moins pour l'heure, indépassable, puisque son secret est dorénavant laissé «à qui s'en pourra servir $»^{46}$. Dans ce cadre très synthétique, Corneille apparaît donc comme un éternel contemporain - n'est-ce pas déjà en faire un « classique »?

Ainsi coexistent au moins deux conceptions différentes du contemporain dans ces divers bilans et examens rétrospectifs du patrimoine littéraire : soit l'on repousse dans le passé les avant-dernières générations pour promouvoir la vitalité de tel ou tel groupe ou de nouvelles valeurs, et alors se dessine un présent toujours ouvert, articulé à l'actualité la plus immédiate et soumis à l'impératif des mises à jour ; soit, ce qui est notamment le fait des Modernes dans le contexte de la Querelle ou de ses prémices, l'on construit un plus grand siècle bénéficiant en revanche des apports des aînés et sans doute plus susceptible d'être articulé à l'histoire politique. Il ne nous était donc pas possible de définir au préalable ce qu'était le contemporain.

\section{ALLER AU DEVANT DE L'HISTOIRE}

Ces différentes tentatives pour inscrire presque immédiatement dans le cours de l'histoire la production contemporaine sont régies par quelques

45. Ibid., pp. 55-56.

46. Bernard de Fontenelle, Histoire du théâtre françois et Vie de Monsieur Corneille, Euvres complètes, op. cit., t. III, p. 95. Sur ce sujet, voir Claudine Poulouin, " Corneille, père de la scène française. La théorisation de la supériorité de Corneille par Fontenelle », XVII ${ }^{e}$ siècle, $\mathrm{n}^{\circ} 225$, octobre 2004, pp. 735-746. 
principes d'écriture qui témoignent non seulement des obligations et des diverses considérations avec lesquelles elles doivent composer, mais encore des spécificités des procédures de consécration qu'elles mettent en scène.

Il est tout d'abord évident que leur intervention dans la production contemporaine se fait sur le mode de la sélection. En effet, se différenciant d'autres types de parcours rétrospectifs qui se donnent notamment pour mission de pérenniser, d'ordonner ou de donner à comprendre un patrimoine menacé par la destruction ou par l'oubli et qui vont donc rechercher l'exhaustivité, ou se différenciant encore des divers périodiques soucieux de transmettre informations ou « nouvelles » complètes, ces bilans relèvent par principe de choix, susceptibles d'orienter le lecteur dans la culture qui caractérise son temps. Ces choix paraissent d'autant plus nécessaires qu'accède désormais au savoir livresque un nouveau public - certains savants comme Baillet ${ }^{47}$ jugent qu'il faut l'armer à cet effet - et qu'en outre est alors réactivée et repensée, comme on l'a montré, la traditionnelle menace de l'《excès des livres » : cette prolifération doit donc être régulée ${ }^{48}$. Ainsi, alors qu'un Colletet avait encore la volonté de couvrir l'ensemble de l'histoire de la poésie nationale, un Sorel en 1667 ne retient que les ouvrages qui ont apporté un " ornement extraordinaire ${ }^{49}$ " à la langue française et c'est encore ici le critère linguistique qui structure la perception du contemporain $^{50}$. D'autre part, alors même qu'il semble difficile à ces hommes, nous l'avons constaté, de parler sereinement des vivants, le report sur le présent d'une activité de sélection précédemment déployée pour le passé, par un glissement presque insensible, permet d'introduire plus aisément des jugements - non seulement parce que le principe de sélection facilite le silence sur quelques noms, mais surtout parce que celui qui estime savoir choisir dans le passé peut se juger également apte à démêler l'écheveau du présent. Sorel, pour sa part, s'autorise de «l'opinion receuë du plus grand nombre de Gens, plutost que la sienne en particulier ${ }^{51} »$. Ainsi se donne-t-il pour l'incarnation d'une conscience collective parvenue à maturité, s'appuyant sur un savoir et des goûts les mieux partagés et les plus nécessaires. La

47. Adrien Baillet, Des jugemens sur les livres [...], op. cit., pp. 2 sq.

48. Voir Jean-Marc Chatelain, "L'excès des livres et le savoir bibliographique ", Littératures classiques, $\mathrm{n}^{\circ} 66$, automne 2008, pp. 145-160.

49. Charles Sorel, op. cit., p. 250.

50. Comme le remarque Michèle Rosellini, la différence du langage d' « aujourd'hui » et de l'ancien le conduit tout d'abord à classer les ouvrages relativement récents selon les matières - précisons que cela n'exclut pas de brefs survols rétrospectifs -, avant de les resituer au sein d'une dernière partie dans une plus large perspective chronologique lui permettant d'observer les progrès de la langue (« La Bibliothèque française de $\mathrm{Ch}$. Sorel : intégration ou liquidation de la bibliothèque humaniste ? », Littératures classiques, $\mathrm{n}^{\circ}$ 66, op. cit., pp. 110-111).

51. Charles Sorel, op. cit., pp. 5-6. Pour une analyse des justifications par Sorel de son droit à exprimer un jugement et à former l'esprit du lecteur et, plus largement, sur l'ensemble de son ouvrage, voir Michèle Rosellini, op. cit., pp. 93-113, notamment les pp. 106-107. 
sélection se présente donc bien moins comme procédant d'une individualité - l'abondance des tournures impersonnelles, notamment, tend à en neutraliser la figure - que comme relevant de la compétence de ceux qui se savent « stilé[s] dans la lecture des bons Livres $»^{52}$ et capables d'exprimer une opinion à valeur coutumière. Dans ces conditions, le « choix de Livres " s'offre comme « raisonné $»^{53}$ et donc propre à pondérer certains aléas de la mode ou du temps. Il paraît alors manifestement légitime à l'auteur de cette « bibliothèque » d'une part de trancher dans le plus récent présent et de ne retenir que quelques-uns des «nouveaux auteurs » de « maintenant $»^{54}$ et, d'autre part, en toute logique, de conférer à sa sélection une dimension non seulement critique, mais encore prospective ${ }^{55}$ : Sorel estime que la traduction de l'Énéide par Segrais ne donnera à celui-ci « pas moins de reputation que ses autres Poësies »; celle de Xénophon par Charpentier lui fait souhaiter que l'écrivain «continuë de s'employer à de semblables travaux » et il espère une traduction de L'Histoire des François de Grégoire de Tours par l'abbé de Marolles ${ }^{56}$. L'élection des meilleurs, se faisant aussi suivi et soutien d'un certain nombre d'auteurs privilégiés, prépare l'avenir : l'effort de raison permet d'ores et déjà d'aller au devant de la postérité.

L'acuité d'une sélection dans la plus récente actualité littéraire est cependant menacée par d'inévitables considérations de hiérarchies sociales et littéraires. Face à cette actualité pour laquelle un classement chronologique des écrivains n'est en outre souvent plus possible, la prudence ou la négligence conditionnent donc parfois le retour au simple principe d'une liste aléatoire, comme le fait Richelet lorsque, après avoir évoqué un premier ensemble de poètes modernes ordonné selon la date respective de leur mort, il en vient à nommer « non pas en Maistre de Cerémonie, mais selon qu'ils s'offrent à [sa] mémoire » les vingt-sept auteurs qui vivent et « travaillent avec réputation $\|^{57}$ : si ce type de liste a l'avantage de souligner la prolifération des écrivains en les rapprochant physiquement dans la page et symboliquement dans le temps, il n'en signifie pas moins ici l'éviction de toute

52. Charles Sorel, op. cit., p. 165.

53. Ibid., p. 273.

54. Au sein du chapitre consacré à la poésie, à propos du théâtre, Sorel passe sans transition des hommes qu'il juge être de la génération de Corneille à ceux qu'il nomme les «nouveaux auteurs », ne retenant que Quinault, Boyer, [Gabriel] Gilbert, Mlle Desjardins, Racine, Scarron (déjà mort à cette date) et Molière, et il se livre également à une sélection des auteurs de « maintenant » dans les domaines de la poésie et de la prose (ibid., pp. 209-213).

55. À cet égard, Karen Abiven et Laure Depretto remarquent que «l'écriture de l'actualité est tiraillée entre plusieurs temporalités, en lien avec le décalage entre le temps de l'action, celui de la rédaction et celui de la publication » (op. cit., p. 11).

56. Charles Sorel, op. cit., pp. 212, 227, 230.

57. Pierre Richelet, La Versification françoise où il est parlé de l'Histoire de la Poésie françoise, des poètes françois anciens et modernes, de l'origine de la rime, et de la manière de bien faire et de bien tourner les vers [...], Paris, Estienne Loyson, 1672, pp. 9-10. 
pensée, même la plus rudimentaire, du temps présent - dans ce cas précis, le recours à la liste témoigne bien des difficultés qu'il peut y avoir à historiciser d'une quelconque manière l'actualité ${ }^{58}$. En revanche, la proximité affichée avec l'auteur dont on parle peut tout à fait être gage d'une valeur et d'une pertinence historiques et donc favoriser l'inscription dans le temps de celui-ci. En effet, alors que pour un Boileau l'écart temporel est perçu comme vecteur de dissipation du faux ${ }^{59}$, pour d'autres il semble susceptible de favoriser les déformations ou les oublis et donc de menacer l'intégrité du jugement. Ainsi l'insertion du témoignage de l'ami ou du parent, notamment au sein des « vies », devient-elle d'autant plus précieuse que, dans le cadre d'une histoire immédiate, elle est moins suspecte d'altérations ${ }^{60}$. C'est aussi que la simple chronique des événements et l'enregistrement des anec$\operatorname{dotes}^{61}$ ne s'opposent pas encore nécessairement à cette époque avec les figures d'un historien ou d'un critique raisonnables, justes et utiles : ils ne sont pas seulement des pratiques mondaines, éventuellement futiles ou accessoires, mais relèvent aussi du désir de transmettre des connaissances, de donner à réfléchir et de travailler pour la postérité.

Dans ce contexte où la contemporanéité n'est pas toujours nécessairement perçue comme un obstacle à la vérité, l'inscription de la production la plus récente au terme d'un plus large procès historique prétend conférer à celle-ci un sens qui la dépasse au sein d'un devenir collectif. D'une part, l'examen du passé offre des fondations à la création contemporaine. C'est le cas notamment des historiques qui remontent à l'Antiquité. En effet, l'évocation des auteurs grecs et romains par laquelle Mademoiselle de Scudéry débute l'histoire de la poésie qu'elle retrace, en 1658, au sein de La Clélie, n'est pas le simple outil d'une reviviscence du motif de la translatio studii, ni la seule mise à disposition du public galant d'un savoir qui peut lui

58. D'autres listes très vastes, émanant de savants, procèdent en revanche à des classements (voir notamment Claude Irson, Nouvelle Methode [...] Avec une liste des Auteurs les plus célebres de nôtre Langue, Paris, G. Meturas, 1656 et Jean-Baptiste de Rocoles, Introduction generale à l'Histoire [...], $3^{\mathrm{e}}$ éd. Paris, D. Bechet, 1664, 2 vol.) ; en ce qui concerne les « annuaires » établis par les mondains, voir Delphine Denis, Le Parnasse galant, op. cit., pp. 36-56.

59. Nicolas Boileau, «Préface » de 1701, dans Euvres complètes, op. cit., p. 3.

60. Donnons juste deux exemples autres que celui de Racan dans sa Vie de Malherbe : dans sa Vie de Monsieur d'Ablancourt, Olivier Patru se prévaut de sa connaissance intime du traducteur pour éclairer, entre six et dix ans après la mort de celui-ci, certaines particularités de son travail, dans le Lucien notamment (voir cette vie et son analyse dans Roger Zuber [1995], op. cit., pp. 419433 ) ; l'auteur de la Vie de Benserade, publiée six ans après la mort du poète, reste anonyme - il s'agit en fait de l'abbé Paul Tallemant -, mais il insiste sur sa proximité avec le poète pour le peindre enjoué et galant à l'image de son œuvre (Discours sommaire de Monsieur L.T. touchant la Vie de M. de Benserade [1 ${ }^{\text {re }}$ éd. 1697], dans Les CEuvres de Monsieur de Benserade, Paris, Ch. De Sercy, 1698, pp. 4, 2, 12 et 24).

61. Sur l'anecdote, nous renvoyons à l'étude de Karen Abiven, " "Comme une anecdote de la veille": mise en scène énonciative de l'actualité dans les genres anecdotiques (1660-1710)», Littératures classiques, $\mathrm{n}^{\circ}$ 78, op. cit., pp. 17-34, notamment pp. 28-29. 
échapper. Elle assure surtout à la production galante et enjouée du milieu du $\mathrm{XVII}^{\mathrm{e}}$ siècle, comme on l'a remarqué, des racines gréco-latines - alors encore essentielles à la durée littéraire -, qui permettent notamment, par les filiations établies avec des auteurs de référence, de distinguer le bon grain de l'ivraie au sein de la production burlesque la plus récente ${ }^{62}$. D'autre part, en retour, c'est évidemment aussi le sens que l'on veut bien attribuer à la création contemporaine qui détermine l'examen du patrimoine. Tout d'abord par des phénomènes d'actualisation et de miroir : Colletet observe d'autant plus les formes de la ballade et du rondeau au Moyen Âge qu'il sait que ses contemporains les ont remises au goût du jour ${ }^{63}$. Mais c'est surtout par la dimension téléologique qu'on lui confère que la création contemporaine donne sens au passé et dévoile son apport à l'histoire collective. Par exemple, c'est en fonction de l'épanouissement du théâtre de son temps et des principes qui le fondent que Colletet perçoit et juge la production de La Peruse $^{64}$. La consécration des contemporains préside également à l'écriture du passé chez Sorel, notamment lorsqu'il reconstruit l'histoire du genre épistolaire en fonction de la lettre familière et élégante de style moyen qu'il entend promouvoir, incarnée par le modèle voiturien : il met en effet successivement de côté, dans la première partie de son propos, toutes les formes d'épîtres puis de lettres qui ne peuvent apparaître comme des ancêtres de ce dernier modèle à l'usage le plus « civil $^{65}$ », avant de se livrer au récit rétrospectif de ce corpus ainsi défini, tout à la cause du triomphe de l'époque moderne.

Ainsi l'assurance de plus en plus affirmée du progrès dont témoigneraient la langue et les lettres contemporaines conduit-elle à amplifier leurs résonances dans l'écriture du passé : à cet égard Fontenelle franchit, dans la dernière décennie du siècle, un pas capital. Son Recueil des plus belles pieces des Poëtes François et surtout son Histoire du théâtre françois et sa Vie de $M$. Corneille montrent que sa perception des bouleversements radicaux qui caractérisent sa propre époque le conduit à relire ce qui les précède à leur lumière et à en chercher l'origine. Il ne s'agit pas simplement par là de prendre acte des évolutions dans le domaine des lettres, ni même d'ordonner le processus historique au travers de son chemin vers la beauté ou la raison, mais, dorénavant, d'en chercher les causes et d'en trouver les

62. Voir ce texte et son analyse par Delphine Denis, dans «De l'air galant» et autres Conversations (1653-1684). Pour une étude de l'archive galante, Paris, Champion, 1998, pp. 183244, notamment pp. 191-193 et 242-244; voir aussi Le Parnasse galant, op. cit., pp. 80-95.

63. Guillaume Colletet, Le Moine de Lire, dans Le Cabinet historique, 1858, t. IV, $1^{\text {re }}$ partie, p. 269 et Jean Meschinot, manuscrit B.N.F., N.A.Fr. 3073, fo 354 recto.

64. Guillaume Colletet, "Jean de La Peruse », Vies [... des] Poëtes angoumoisins, op. cit., pp. 212-216. Ce sont notamment les règles de bienséance que La Peruse « ne sçavoit pas encore » (p. 215).

65. Charles Sorel, op. cit., pp. 106-114 sq. 
« principes généraux ${ }^{66}$ ». Ainsi non seulement cette réflexion sur les causes des transformations se poursuit-elle au sein de la période contemporaine elle-même ${ }^{67}$, mais elle conduit surtout Fontenelle à déterminer, notamment au travers du cas de Corneille, ce qui constitue la modernité littéraire : il entend alors montrer, comme on le sait, qu'elle procède des progrès dans l'art de penser. Dans ce cadre, il s'agit donc moins de se livrer à la consécration des meilleurs auteurs des temps actuels que de dévoiler ce qui peut en être légitimement à la source, ce qui peut la motiver et la valider, et cet effort de dévoilement est bien fait in fine, comme il le dit de son Recueil, « pour le temps présent ${ }^{68}$ ». En bref, on constate que n'est déjà plus très lointain le moment où $l^{1}$ ' " histoire de l'esprit humain ${ }^{69}$ » va à nouveau transformer les rapports entre la compréhension du passé et l'examen du présent.

$\mathrm{Au} \mathrm{XVII}{ }^{\mathrm{e}}$ siècle, la détermination d'une actualité littéraire ne dépend donc que partiellement de la vie des individus qui en seraient les acteurs : son découpage semble plutôt relever de la nouveauté des sens ou des valeurs que l'on veut bien attribuer à la langue, aux œuvres et aux pratiques susceptibles de la caractériser. Il est donc logique que ce soit majoritairement les Modernes, ou ceux qui leur ouvrent la voie d'une manière ou d'une autre, qui entreprennent d'historiciser la production qui leur est contemporaine même si, comme on le sait, cela ne signifie pas pour autant que les Anciens n'aient pas aussi vive conscience de la singularité et de la créativité de leur temps.

Quels qu'ils soient cependant, ces bilans représentent une forme d'intervention dans un présent face auquel toute inquiétude n'est pas nécessairement bannie et que l'on place encore, dans tous les cas, sous l'œil de la postérité. Cette intervention procède en effet non seulement du désir d'orienter le lecteur au sein d'une production jugée trop abondante et inégale, mais également du désir de conférer à cette production elle-même des orientations, aussi diverses puissent-elles être. Dans cette perspective, l'octroi aux œuvres récentes de racines et de passés, éloignés ou proches, se fait chemin de rencontre de la postérité : sans doute espère-t-on par là même orienter également le jugement des siècles à venir.

66. Bernard de Fontenelle, Sur l'histoire, Euvres complètes, op. cit., t. III, pp. 177-178.

67. C'est par exemple le cas lorsque Fontenelle, s'efforçant de comprendre comment le « bon temps » a pu passer, recherche la «liaison nécessaire, mais cachée » qui règle le changement insensible des goûts entre la génération de Voiture et celle qui lui succède (ibid., pp. 183-185 ; voir aussi la Digression sur les anciens et les modernes, Euvres complètes, op. cit., t. II, p. 429).

68. Bernard de Fontenelle, «Préface " du Recueil des plus belles pieces des Poëtes François, op. cit., p. 190.

69. Voir Jean Dagen, L'Histoire de l'esprit humain dans la pensée française de Fontenelle à Condorcet, Paris, Klincksieck, 1977. 
Il n'est pas certain de fait que ces guides n'aient pas réussi, dans une certaine mesure, à pérenniser - pour un temps du moins - l'ordre et les raisons qu'ils confèrent, si promptement, à la production de leur temps : on sait notamment que la première réception est souvent déterminante et que parfois les schèmes, jugements et autres caractérisations se fixent et se propagent avant qu'ils ne puissent faire l'objet de diverses et véritables réévaluations. La détermination du "classicisme" est peut-être plus précoce qu'on ne le croit - sans doute sa capacité à s'imposer est-elle aussi constitutive de son identité. 